\title{
Variabilidad en la abundancia relativa, estructura por tallas y proporción de sexos del dorado Coryphaena hippurus (Pisces: Coryphaenidae) en el Golfo de Tehuantepec, México
}

\author{
Carmen Alejo-Plata ${ }^{1}$, José Luis Gómez ${ }^{2} \&$ Saúl J. Serrano-Guzmán ${ }^{3}$
}

1, 3. Universidad del Mar, Campus Puerto Ángel, Ciudad Universitaria, Puerto Ángel, San Pedro Pochutla, C.P: 70902, Oaxaca, México; plata@angel.umar.mx,sserrano@angel.umar.mx

2. Facultad de Estudios Superiores Zaragoza, Universidad Nacional Autónoma de México, Av. 5 de Mayo y Fuerte de Loreto, Col. Ejército de Oriente, Iztapalapa, Apartado Postal 09230 CP 9-020, México D.F., México; lgomez@servidor.unam.mx

\author{
Recibido 22-VII-2013. Corregido 10-XII-2013. Aceptado 17-I-2014.
}

\begin{abstract}
Variability in the relative abundance, size structure and sex ratio of the dolphinfish Coryphaena hippurus (Pisces: Coryphaenidae) in the Gulf of Tehuantepec, México. The dolphinfish (Coryphaena hippurus), is an oceanic epipelagic fish found worldwide in tropical and subtropical waters, with a high dispersal capability via large-scale migrations. This fast-swimming top-level predator is abundant in the Gulf of Tehuantepec, where it is caught incidentally by artisanal fisheries, and represents a target species for both recreational and commercial fisheries in Mexico, Ecuador, Peru and Central America. Nowadays, local fishery information on this species is scarce, thus our objective was to analyze the size structure by sex and the catchper-unit-effort (CPUE) tendency of dolphinfish caught in the Gulf of Tehuantepec, from 2000 to 2007. For this, fishery catches information was obtained from the artisanal fleet, at six landing sites in the Gulf, and the sex ratio, fork length (FL) and the catch per unit effort (CPUE) were estimated. From all sampling sites, a total of 3494 females, and 3877 males were obtained, and dolphinfish size as fork length (FL) ranged from 20.5 to $152 \mathrm{~cm}$. Fish size ranged from 25.5 to $148 \mathrm{~cm}$ furcal length (FL) in males, and 20.5 to $129 \mathrm{~cm}$ FL in females. The sex ratio (males:females) was 1:1, except in April-May $(1: 1.5, \mathrm{p}<0.05)$ and November $(1: 0.5, \mathrm{p}<0.05)$. The sex ratio at different size classes showed a significant bias towards females at smaller sizes $(<75 \mathrm{~cm} \mathrm{FL})$, whereas the males were predominant in larger size classes $(>100 \mathrm{~cm} \mathrm{FL})$. The size structure was bimodal, with a variation in the size average; the modes were defined as the small group $(\mathrm{FL}=50-55 \mathrm{~cm})$ and the large size group $(\mathrm{FL}=100-110 \mathrm{~cm})$. The CPUE showed seasonal changes: values were high for the November-December period, and values were lower for July-August. The seasonal and inter annual variation in the abundance of dolphinfish is probably related to a pre-spawning migration in close relation to the rain-drought regime characteristic of the region, and the associated wind upwelling season of "Tehuanos" in the Gulf of Tehuantepec. Rev. Biol. Trop. 62 (2): 611-626. Epub 2014 June 01.
\end{abstract}

Key words: Mahi mahi, dolphinfish, artisanal fishing, size structure, CPUE, Eastern Tropical Pacific.

Coryphaena hippurus, también conocido como "dorado", "mahi-mahi" o "dolphinfish" es un depredador tope epipelágico migratorio, que se distribuye en aguas tropicales y subtropicales de todos los océanos. Es una especie de rápido crecimiento, llega a medir más de dos metros y pesar hasta $30 \mathrm{~kg}$; alcanza su madurez sexual alrededor de los $50 \mathrm{~cm}$ de longitud furcal (Palko, Beardley \& Richards, 1982). El dorado soporta pesquerías comerciales y deportivas; la magnitud y estabilidad de los niveles de captura, sugiere que es un recurso resiliente que es capaz de soportar altas tasas de captura y adaptarse a cambios ambientales (Campos, Segura, Lizano \& Madrigal, 1993; Mahon \& Oxenford, 1999; Massutí, Morales-Nin \& Moranta, 1999; Oro, 1999; Zúñiga, Ortega-García \& KlettTraulsen, 2008). 
Este recurso ha aportado volúmenes importantes a la captura pesquera en el Mediterráneo, Caribe, Pacífico Tropical Oriental y EE. UU. (Patterson \& Martínez, 1991; Norton \& Crooke, 1994; Massutí \& Morales-Nin, 1995; Arocha, Marcano, Láraez, Altuve \& Alío, 1999; Lasso \& Zapata, 1999; Mahon \& Oxenford, 1999; Schwenke \& Buckel, 2008). En el Pacífico mexicano, se han reportado abundancias importantes de dorado desde el Golfo de Tehuantepec hasta el sur de la Península de Baja California y al interior del Golfo de California (Santana-Hernández, 2001). En México, esta especie junto con los peces de pico (pez vela, Istiophorus platypterus; marlín azul, Makaira indica; marlín negro, Makaira mazara) están reservados por ley para la pesca recreacional dentro de los $96.2 \mathrm{~km}$ de la costa (Diario Oficial de la Federación, 1995).

En el Golfo de Tehuantepec la pesca deportiva no es una actividad constante y se limita a tres torneos anuales (mayo, octubre y noviembre), por lo que la información biológica que se puede obtener de esta actividad no es suficiente para proporcionar un diagnóstico pesquero de $C$. hippurus. En esta región, sin embargo, el dorado es una parte importante de la captura de la flota artesanal, que opera con permisos para tiburón y peces demersales; esta captura artesanal ha permitido dar seguimiento y generar información sobre el estado del recurso. Tomando en consideración el crecimiento de las actividades económicas y sociales de los habitantes de la zona en estudio, y la necesidad de contar con información que pueda contribuir a la elaboración de un esquema más adecuado para el manejo de la pesquería del dorado, en este estudio se presenta la descripción de la tendencia de la abundancia relativa, estructura de longitudes y proporción de sexos del $C$. hippurus, capturado durante el periodo de 2000 a 2007, por la flota artesanal en el Golfo de Tehuantepec, México.

\section{MATERIAL Y MÉTODOS}

Área de estudio: El Golfo de Tehuantepec es un área tropical del sureste de México, con un alto potencial pesquero, debido a sus características fisiográficas y batimétricas, que en combinación con la estacionalidad de procesos atmosféricos y oceanográficos lo hacen mantener una productividad relativamente alta prácticamente a lo largo del año (Gallegos-García \& Barberán-Falcón, 1998; Lara-Lara, Robles-Jarero, Bázan-Guzmán \& Millan-Núnez, 1998), que favorecen el desove de diferentes especies. Esto lo convierte también en un lugar de crianza y alimentación de organismos marinos de interés comercial; y una área de importantes pesquerías como camarón, atún y sardina entre otras especies (Ortega-García, Trigueros-Salmerón, Rodríguez-Sánchez, Lluch-Cota \& Villalobos, 2000).

La región del Pacífico sur mexicano, que incluye al Golfo de Tehuantepec y áreas adyacentes, transita a lo largo del año por dos épocas o temporadas climáticas. Una, la temporada de lluvias y tormentas tropicales, desde finales de mayo o principios de junio hasta finales de septiembre o principios de octubre; que coincide con los meses "cálidos", donde frente a las costas de Oaxaca y Chiapas se localiza una de las zonas generadoras de perturbaciones tropicales más activas del mundo (Lluch-Cota, Álvarez-Borrego, Santamaría del Ángel, Mueller-Karger \& Hernández-Vázquez, 1997). La otra, la época de estiaje, coincide con los meses "frescos", desde finales de octubre a principios de noviembre y hasta finales de abril o principios de mayo; es la temporada en que los frentes fríos que se desplazan desde Canadá y Estados Unidos hacia el Golfo de México provocan "nortes" a medida que descienden y se intensifican produciendo vientos de chorro o eventos de viento intensos y de baja altitud sobre el Istmo de Tehuantepec (Clarke, 1988; McCreary, Lee \& Enfield, 1989; Lavin et al., 1992; Romero-Centeno, Zavala-Hidalgo, Gallegos \& O’Brien, 2003). Estos se dirigen en pulsos intermitentes de gran intensidad hacia el océano Pacífico, provocando lo que localmente se conoce como Tehuanos o Tehuantepecanos; que inducen surgencias costeras en el Golfo de Tehuantepec, así como giros ciclónicos de mediana escala que se intensifican por 
advección derivando hacia el occidente y anticiclónicos de menor magnitud e intensidad hacia el oriente, que en su mayoría desaparecen por la mezcla vertical (Roden, 1961; MonrealGómez \& Salas de León, 1998; Chelton, Freilich \& Esbensen, 2000a,b; González-Silveira, Santamaría del Ángel, Millán-Núnez \& Manzo-Monroy, 2004; Trasviña \& Barton, 2008).

Trabajo e información de campo: De diciembre 2000 a diciembre 2007 se efectuaron recolectas en seis sitios de desembarco de la flota artesanal en el Golfo de Tehuantepec $\left(13^{\circ} 30^{\prime} 16^{\circ} 30^{\prime} \mathrm{N}-92^{\circ} 30^{\prime} 6^{\circ} 00^{\prime} \mathrm{W}\right)$. El trabajo de campo se realizó durante dos días a la semana en las localidades de Puerto Ángel, Puerto Escondido y Huatulco; y mensualmente en Punta Chipehua, Boca del Cielo y Puerto Chiapas. Las zonas de pesca están localizadas entre 9.3-92.6km mar afuera.

La pesca artesanal de pelágicos mayores en Puerto Ángel estuvo conformada por 228 embarcaciones menores de fibra de vidrio de 7.61 a $10.33 \mathrm{~m}$ de eslora, con una capacidad de carga de entre 1200 y $3000 \mathrm{~kg}$ y con motor fuera de borda de 40 a $75 \mathrm{HP}$. Las operaciones de pesca diarias iniciaron entre 6:00 y 7:00 de la mañana y tuvieron una duración de cuatro a cinco horas, y se realizaron con equipos construidos de manera artesanal, donde participan de dos a cuatro pescadores.

Los equipos de pesca activos que se utilizan son los "curricanes", para la captura del barrilete negro (Euthynnus lineatus), atún aleta amarilla (Thunnus albacares) y juveniles de dorado; el palangre modificado con boyas para la captura de pez vela (I. platypterus), dorado (C. hippurus) y tiburón (de varias especies). Los equipos pasivos son los palangres (cimbras) y las redes de enmalle, los cuales son utilizados para la pesca de tiburón (Cuadro 1).

A cada ejemplar de dorado se le registró con una cinta métrica la longitud furcal (Lf, con una precisión de $\pm 0.5 \mathrm{~cm}$ ) y el peso

CUADRO 1

Descripción de las unidades de pesca utilizadas en la pesca artesanal en el Golfo de Tehuantepec, México

TABLE 1

Description of fishing units used in artisanal fisheries in Gulf of Tehuantepec, México

\begin{tabular}{|c|c|c|c|}
\hline Unidad de pesca & Especies objetivo & Equipos de pesca & Especies incidentales \\
\hline $\begin{array}{l}\text { Demersal } \\
\text { Lanchas con motor } \\
\text { fuera de borda } \\
7.5 \mathrm{~m} \text { eslora/ canoas } \\
\text { de madera sin } \\
\text { motor }\end{array}$ & Peces óseos demersales & $\begin{array}{l}\text { - Redes de enmalle } \\
3 \text { a } 5 ", \text { longitud } 100 \mathrm{~m}\end{array}$ & $\begin{array}{l}\text { Tiburón (juveniles } \\
\text { y neonatos), rayas, } \\
\text { C. hippurus Lf } \\
<20<60 \mathrm{~cm}\end{array}$ \\
\hline $\begin{array}{l}\text { Pelágica } \\
\text { Lanchas con motor } \\
\text { fuera de borda } \\
7.5 \mathrm{~m} \text { eslora }\end{array}$ & $\begin{array}{l}\text { Atún (Tunus albacares), } \\
\text { barriletes (Euthynus } \\
\text { linneatus, Katsuwonus } \\
\text { pelamis) }\end{array}$ & $\begin{array}{l}\text { - Curricanes, anzuelos dobles } \\
\text { No. } 24 \text { o } 25 \\
\text { - Palangre de superficie } \\
\text { anzuelo noruego recto No. } 1 \text { ó } 0 \text {, garra de } \\
\text { águila } 14 / 0 \text { a } 16 / 0 \\
\text { - Palangre de superficie con boyas } \\
\text { anzuelo noruego recto No. } 1 \text { ó } 0 \text {, garra de } \\
\text { águila } 14 / 0 \text { a } 16 / 0 \text {; carnada viva }\end{array}$ & $\begin{array}{l}\text { Tiburón (juveniles), } \\
\text { I. platypterus, C. } \\
\text { equiselis, C. hippurus } \\
\mathrm{Lf}>60 \mathrm{~cm} .\end{array}$ \\
\hline $\begin{array}{l}\text { Tiburonera } \\
\text { Lanchas con motor } \\
\text { fuera de borda, } \\
\text { 10m eslora }\end{array}$ & $\begin{array}{l}\text { Tiburón (C. falciformis, } \\
\text { C. limbatus, C. leucas, } \\
\text { S. lewini, A. Pelagicus, } \\
\text { A. vulpinus, N. velox, } M \text {. } \\
\text { lunulatus, C. porosus, G. } \\
\text { cuvier, R. lungurio) }\end{array}$ & $\begin{array}{l}\text { - Palangre de superficie } \\
\text { anzuelo noruego recto } 1 \text { ó } 01 \text {, garra águila } \\
16 / 0 \text {, japonés } \\
\text {-Redes de deriva } \\
8 \text { a } 12 \text { ", longitud hasta } 300 \mathrm{~m} \\
\text { - Palangre de superficie con boyas }\end{array}$ & $\begin{array}{l}\text { I. platypterus, } C \text {. } \\
\text { equiselis, C. hippurus } \\
\text { Lf }>90 \mathrm{~cm} \text {. }\end{array}$ \\
\hline
\end{tabular}


total ( $\mathrm{P}$, con una precisión de $\pm 0.01 \mathrm{~kg}$ ) con una balanza digital. El sexo se reconoció por la presencia de una pronunciada cresta ósea en los machos maduros, pero ausente en las hembras (Collete, 1995); y en los peces jóvenes por el examen macroscópico de las gónadas al momento de eviscerarlos. La madurez gonádica se asignó mediante la observación macroscópica de las gónadas con los criterios establecidos por Alejo-Plata, Díaz-Jaimes \& Salgado-Ugarte, (2011).

La información del índice de abundancia relativa (CPUE), provino exclusivamente de las capturas de la flota artesanal con base en Puerto Ángel Oaxaca. De junio de 2005 a diciembre del 2007 se registró la captura y el esfuerzo de pesca de toda la flota artesanal. El trabajo en campo se realizó durante dos días a la semana, donde se registró el total de viajes de pesca de pelágicos mayores.

Índice de abundancia relativa: Para estimar la CPUE, se consideró como unidad de esfuerzo un viaje de pesca. En la pesca artesanal de la costa de Oaxaca, el tiempo para dirigirse al área de pesca fue relativamente corto, debido a que los viajes son diarios y de poca duración. Al respecto, González-Becerril, Espino-Barr, Cruz-Romero \& Ruíz-Luna. (2000) y Arocha, Ortíz, Barrientos, Debrot \& Marcano. (2009, 2012) consideran que las prácticas de pesca de este tipo son suficientemente homogéneas, lo que permite considerar al viaje de pesca como la unidad de esfuerzo. Así la CPUE fue definida como sigue:

$$
C P U E=\frac{\sum_{i=1}^{n} C}{\sum_{i=1}^{n} f}
$$

donde, CPUE es el número de organismos capturados por viaje de pesca; $C$ es el número de organismos; $f$ es el número de viajes y $n$ es el número de operaciones de pesca durante los días de pesca. Para lograr una estimación del promedio de la CPUE se usó el modelo general de Pennington (1996).
Estructura de Lf y proporción de sexos: La distribución de Lf para cada muestra fue analizada usando el estimador de densidad por Kernel (EDK), método estadístico originalmente propuesto por Rosenblatt (1956) y definido como:

$$
\hat{f}(x)=\frac{1}{n h} \sum_{i=1}^{n} k\left(\frac{x-X_{i}}{h}\right)
$$

dónde: $\hat{f}(x)=$ estimación de la variable $x ; n$ $=$ número de observaciones; $h=$ amplitud de banda (intervalo); $X_{i}=$ longitud del i-esimo espécimen (dorado); $k=$ función ponderal Kernel.

Fue utilizada la función Kernel Gaussiana:

$$
\begin{gathered}
K_{z}=\frac{1}{\sqrt{2 \pi}} \exp \left(-\frac{z^{2}}{2}\right) \\
z=\frac{x-X_{i}}{h}
\end{gathered}
$$

Como valor de referencia, la amplitud de intervalo $(h)$ fue determinada con base en la regla para la amplitud óptima de Silverman (1986):

$$
h=\frac{0.9 \mathrm{~A}}{n^{1 / 5}}
$$

dónde:

$$
A=\left[\left(\frac{\sum\left(X_{i}-x\right)^{2}}{n-1}\right)^{1 / 2},\left(\frac{H \text { dispersión }}{1.349}\right)\right]
$$

$H$ dispersión: Dispersión de los cuartos sensu Tukey, 1977; Velleman \& Hoaglin, 1981; Hoaglin, 1983; Fox 1990 (vide in Salgado-Ugarte, 1992, 2002), aproximadamente igual al recorrido intercuartílico (Stata Corporation, 2012).

Para determinar el número y valor de las modas en cada distribución, así como para seleccionar la amplitud de banda adecuada, se 
empleó una versión computarizada de la prueba de multimodalidad (Silverman, 1986), sobre la base de un esquema de toma de muestras con repetición (bootstrap suavizado) escrita para el paquete estadístico Stata (SalgadoUgarte, Shimizu \& Taniuchi, 1994, 1997: Stata Corporation, 2012). En esta prueba, el número de modas queda indicado por el primer valor de significancia ( $\mathrm{p} \geq 0.4$, Salgado-Ugarte et al., 1997).

Se convirtieron a frecuencias absolutas las distribuciones multimodales de densidad obtenidas del paso anterior, para determinar y caracterizar cada uno de los componentes individuales de la distribución mezclada, por medio de una versión computarizada del método semi-gráfico de Bhattacharya (1967), disenado para estimar los componentes gaussianos considerando porciones bien separadas de los componentes en distribuciones mezcladas (Salgado Ugarte et al., 1994; Salgado Ugarte Gómez-Márquez \& Peña-Mendoza, 2005).

La variación del promedio de la Lf durante todo el periodo de estudio, se analizó mediante un análisis de varianza de una vía (ANOVA I, $\mathrm{p}<0.05$ ) (Zar, 1999). La proporción de sexos, estuvo basada en la razón hembras/machos de la muestra analizada. Se utilizó el análisis de Chi cuadrada para determinar si la proporción de sexos por intervalo de longitud y por mes se desviaba de la relación 1:1 (Zar, 1999).

\section{RESULTADOS}

Abundancia relativa: Durante el periodo de estudio, se registró un total de 12241 viajes de pesca. En número de organismos, las capturas pelágicas de la pesca artesanal estuvieron compuestas principalmente por Euthynus lineatus (barrilete negro: 69\%); Sarda orientalis bonito: 8\%); Thunnus albacares (atún aleta amarilla: 8\%); Istiophorus platypterus (pez vela: 4\%); Coryphaena hippurus (dorado: $8 \%$ ); mientras que los tiburones, principalmente Carcharhinus falciformis (tiburón sedoso) aportaron el $3 \%$ a las capturas.

La serie de tiempo del índice de abundancia relativa estimado para C. hippurus se muestra en la figura 1; la variabilidad interanual no presentó diferencias significativas $\left(\mathrm{F}_{(2,33)}=0.8239, \mathrm{p}=0.4475\right)$.

El esfuerzo de pesca promedio mensual tuvo diferencias altamente significativas durante los años analizados: 2005 (Kruskal-Wallis, $\mathrm{H}_{(11,3,220)}=610.6666, \mathrm{p}=0.00$ ); 2006 (KruskalWallis, $\left.\mathrm{H}_{(11,3,582)}=165.4198, \mathrm{p}=0.00 \mathrm{p}<0.001\right)$ y 2007 (Kruskal-Wallis, $\mathrm{H}_{(11,5,439)}=1144.125$, $\mathrm{p}=0.00$ ).

La captura promedio más alta se registró consistentemente en noviembre-diciembre, seguida de mayo (13.1 peces/viaje) y la más baja en agosto (0.05 peces/viaje). Durante el periodo de estudio se observó una tendencia estacional de la abundancia relativa del dorado, los valores más bajos se observaron consistentemente durante los meses de junio-agosto, luego fueron incrementando hacia los meses de invierno (noviembre a enero) y volvieron a decaer durante febrero-marzo, para incrementar nuevamente antes de la temporada de lluvias (abril a mayo).

Estructura de tallas y proporción de sexos: La distribución de frecuencias de la Lf de C. hippurus capturado por la flota artesanal del Golfo de Tehuantepec durante el periodo de 2000 a 2007 estuvo basada en 7371 mediciones de Lf (Fig. 2). Se registraron 3494 hembras en un intervalo de tallas de 20.5 a $152.0 \mathrm{~cm} \mathrm{Lf}$ (Media $=79.32 \mathrm{~cm}, \mathrm{DE}=23.75 \mathrm{~cm}$ ) y pesos de 0.1 a $17 \mathrm{~kg}($ Media $=2.82 \mathrm{~kg}, \mathrm{DE}=3.18 \mathrm{~kg}) ; 3877$ machos en el intervalo de 25.0 a $152.0 \mathrm{~cm} \mathrm{Lf}$ Media $=95.59 \mathrm{~cm}, \mathrm{DE}=22.92 \mathrm{~cm}$ ) y pesos de 0.2 a $31.3 \mathrm{~kg}$ (Media=5.82kg, DE=5.58kg). Durante el periodo de estudio se observaron cambios mensuales en la proporción de sexos y en la distribución mensual de Lf (Fig. 3, 4).

La distribución mensual de $\mathrm{Lf}$ del dorado sugiere que, en mayo a julio los cardúmenes están compuestos sólo por peces pequeños $<75 \mathrm{cmLf}$, y una proporción de sexos $1 \mathrm{M}: 1.5$ $\mathrm{H}$ significativamente distinta de la igualdad $\left(\chi_{0.05}^{2}=51.67, \mathrm{p}<0.05\right)$. En mayo se determinaron cuatro modas con tallas entre 35 y $75 \mathrm{~cm}$ Lf; en junio y julio sólo se detectó una moda con organismos menores a $70 \mathrm{~cm} \mathrm{Lf}$; a partir 

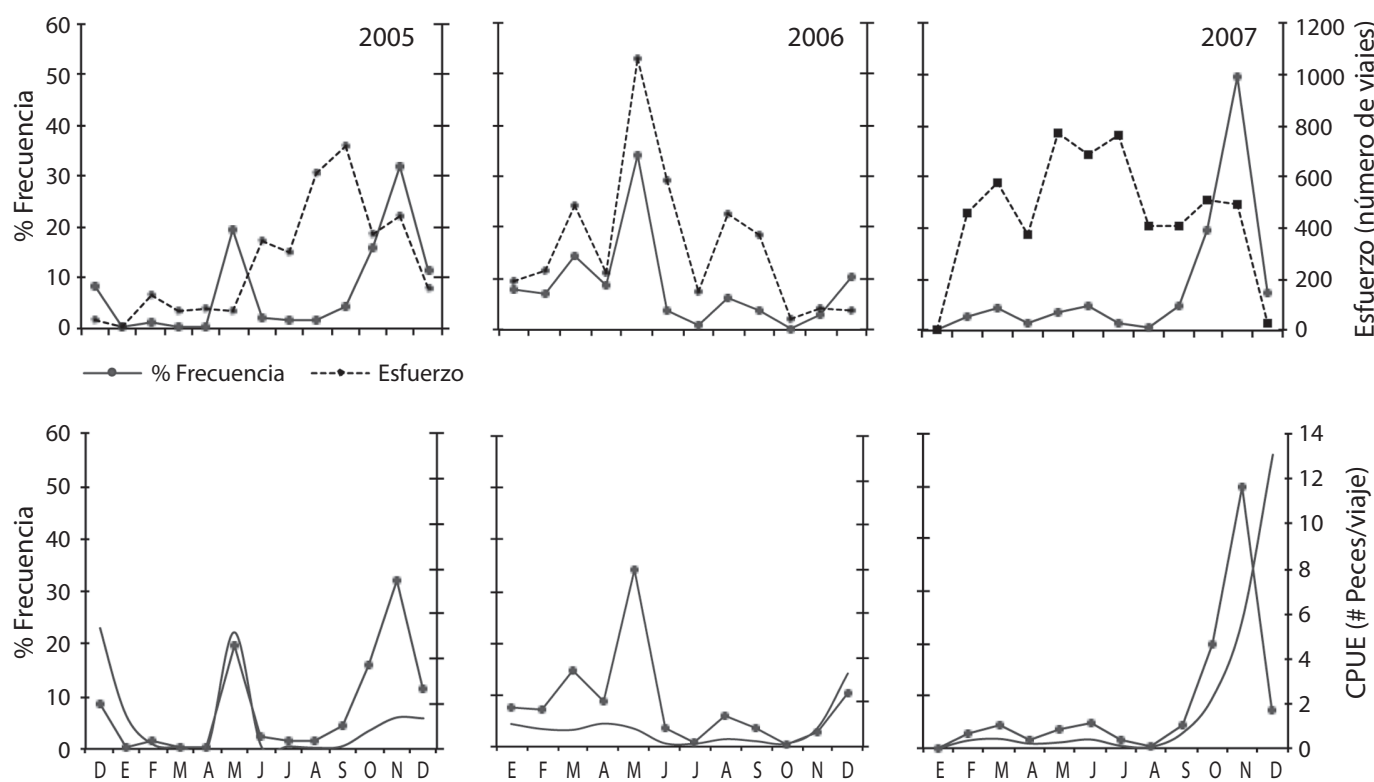

$\rightarrow \%$ Frecuencia — Esfuerzo

Fig. 1. Tendencias de variación entre la frecuencia de captura, esfuerzo de pesca y CPUE de Coryphaena hippurus capturado por la flota artesanal en el Gofo de Tehuantepec durante el periodo 2005-2007.

Fig. 1. Trends of variation among capture frequency, fishing effort and CPUE for Coryphaena hippurus caught by the artisanal fishery in the Gulf of Tehuantepec from 2005-2007.

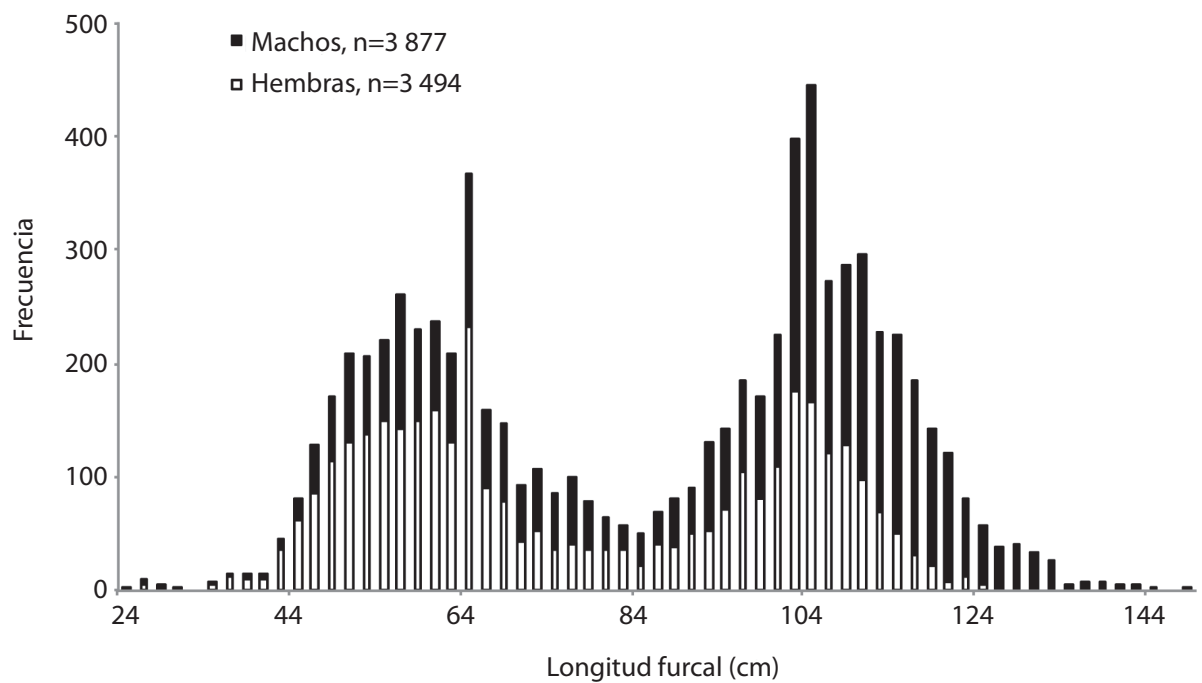

Fig. 2. Distribución de frecuencias de longitud para hembras y machos de Coryphaena hippurus, capturados por pesca artesanal en el Golfo de Tehuantepec. Datos recolectados de 2000 a 2007.

Fig. 2. Frequency distribution of length for males and females of Coryphaena hippurus, caught by the artisanal fishery in the Gulf of Tehuantepec. Data collected from 2000 to 2007. 


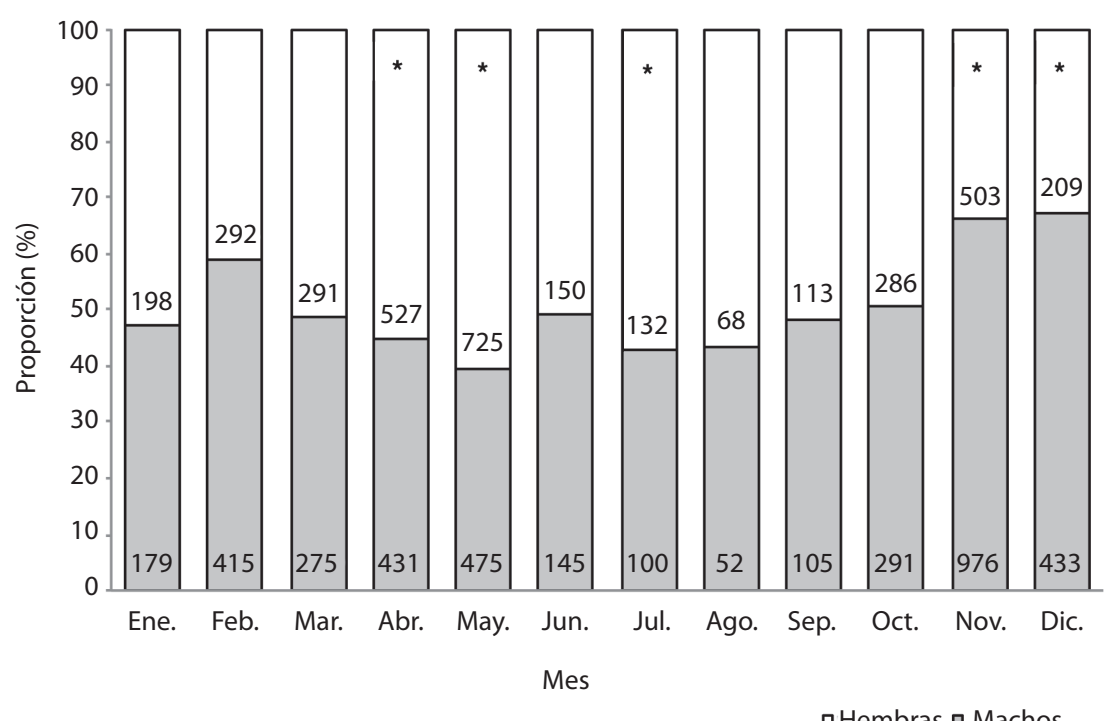

Fig. 3. Proporción de sexos por mes para Coryphaena hippurus, el número sobre la barra corresponde al tamaño de muestra analizado. Datos recolectados de diciembre 2004 a diciembre 2007, en el Pacífico Sur de México. * indica diferencias significativas en la proporción de sexos.

Fig. 3. Monthly sex ratio, the number inside the bars corresponds to the number of sampled fish. The data were collected from December 2004 to December 2007. *significant differences statistical in the sex ratio indicated.

de agosto se incorporaron dorados con tallas mayores a $100 \mathrm{~cm}$ Lf, observándose una disminución en la proporción de hembras (1 M: $\left.1.3 \mathrm{H} ; \chi_{0.05}^{2}=1.88, \mathrm{p}<0.05\right)$ y una distribución bimodal. Entre septiembre y abril la captura estuvo conformada por un amplio intervalo de tallas (23 a $149 \mathrm{~cm}$ Lf), y de cuatro a seis grupos modales. Al inicio de la temporada de secas, noviembre y diciembre, se observó una mayor proporción de machos, 2 a $3 \mathrm{M}: 1 \mathrm{H}$ $\left(\chi_{0.05}^{2}=150.63, \mathrm{p}<0.05 ; \chi_{0.05}^{2}=78.27, \mathrm{p}<0.05\right.$, respectivamente) con tallas $>100 \mathrm{~cm} \mathrm{Lf.} \mathrm{La}$ proporción de sexos tuvo patrones estacionales a través del año (Fig. 3).

Los organismos juveniles con $\mathrm{Lf}<25 \mathrm{~cm}$ no presentan dimorfismo sexual externo, éste se hizo evidente a partir de los $35 \mathrm{~cm}$ de Lf. La proporción de sexos mostró diferencias con la Lf, para longitudes menores a $50 \mathrm{~cm}$ las hembras conforman el $73 \%$ de las capturas ( $1 \mathrm{M}$ : $\left.2.72 \mathrm{H} ; \chi_{0.05}^{2}=53.19, \mathrm{p}<0.05\right)$; en el intervalo de tallas de 55 a $100 \mathrm{~cm}$ Lf las capturas estuvieron compuestas por $63 \%$ de hembras ( $1 \mathrm{M}$ : $\left.1.67 \mathrm{H} ; \chi_{0.05}^{2}=26.65, \mathrm{p}<0.05\right)$. A tallas mayores a $100 \mathrm{~cm}$ Lf los machos predominaron, con un $66.4 \%$ ( $1 \mathrm{M}: 0.5 \mathrm{H} ; \chi_{0.05}^{2}=361.97, \mathrm{p}<0.05$ ).

Coryphaena hippurus presentó un amplio intervalo de Lf en ambos sexos; no obstante, las mayores capturas se observaron sobre los grupos modales de $50-55$ y de 100 a $110 \mathrm{~cm}$ de Lf; el grupo modal entre 80 y $90 \mathrm{~cm}$ Lf fue el menos abundante ( $\sim 5 \%)$ (Fig. 4).

\section{Variabilidad interanual de la estructura} de tallas: $\mathrm{Al}$ ordenar las tallas promedio con respecto al tiempo, se observó una variabilidad interanual en la talla media, de machos y hembras durante el periodo 2000-2007. Los resultados del análisis de varianza muestran diferencias significativas $\left(\mathrm{F}_{(2,33)}=0.8239\right.$, $\mathrm{p}=0.4475$ ) en las tallas medias según el sexo para todos los años considerados.

En 2002 se presentaron las mayores tallas promedio, $99.9 \mathrm{~cm}$ en hembras y $106.24 \mathrm{~cm} \mathrm{Lf}$ en los machos; a partir del 2003 la tendencia fue negativa, los menores valores se observaron en el 2006 con $74.41 \mathrm{~cm}$ para las hembras y $92.36 \mathrm{~cm}$ Lf en machos. Hacia el 2007 se 

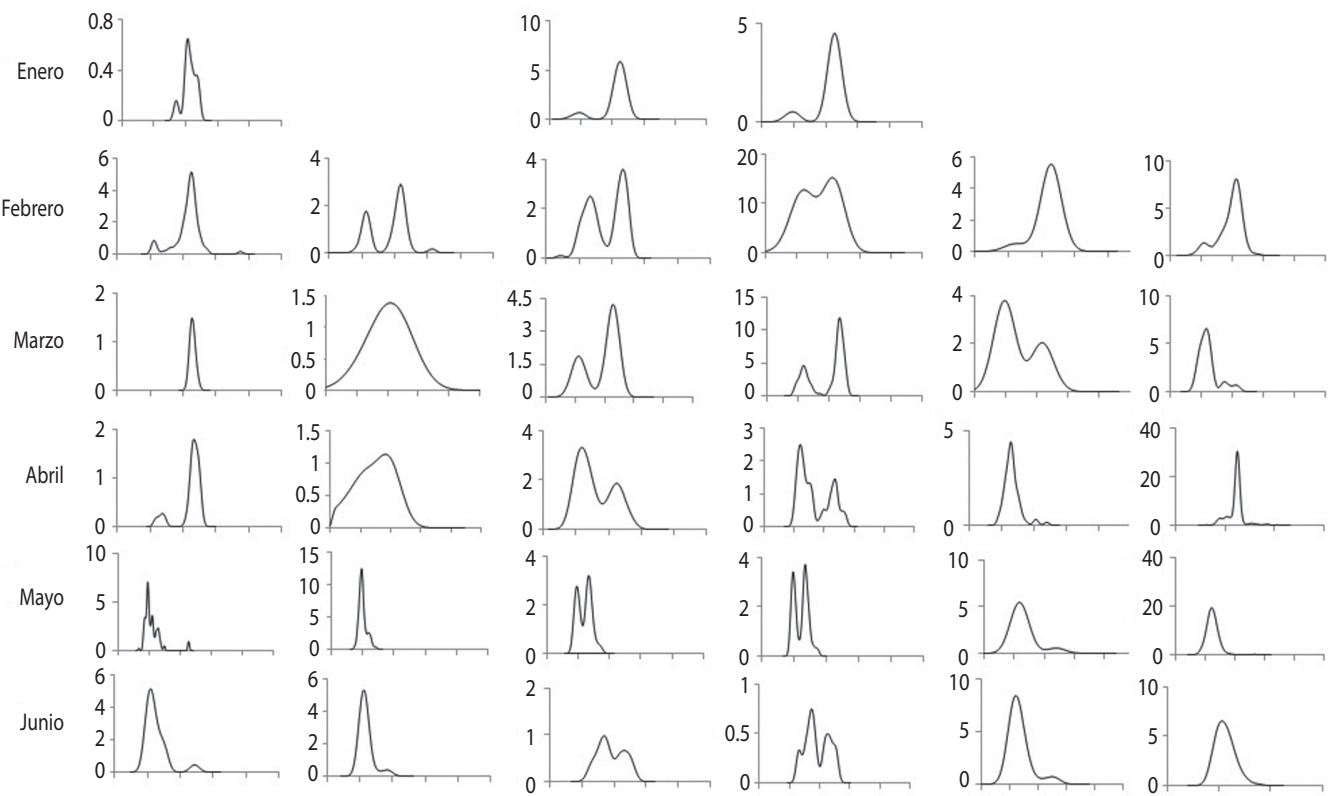

Junio
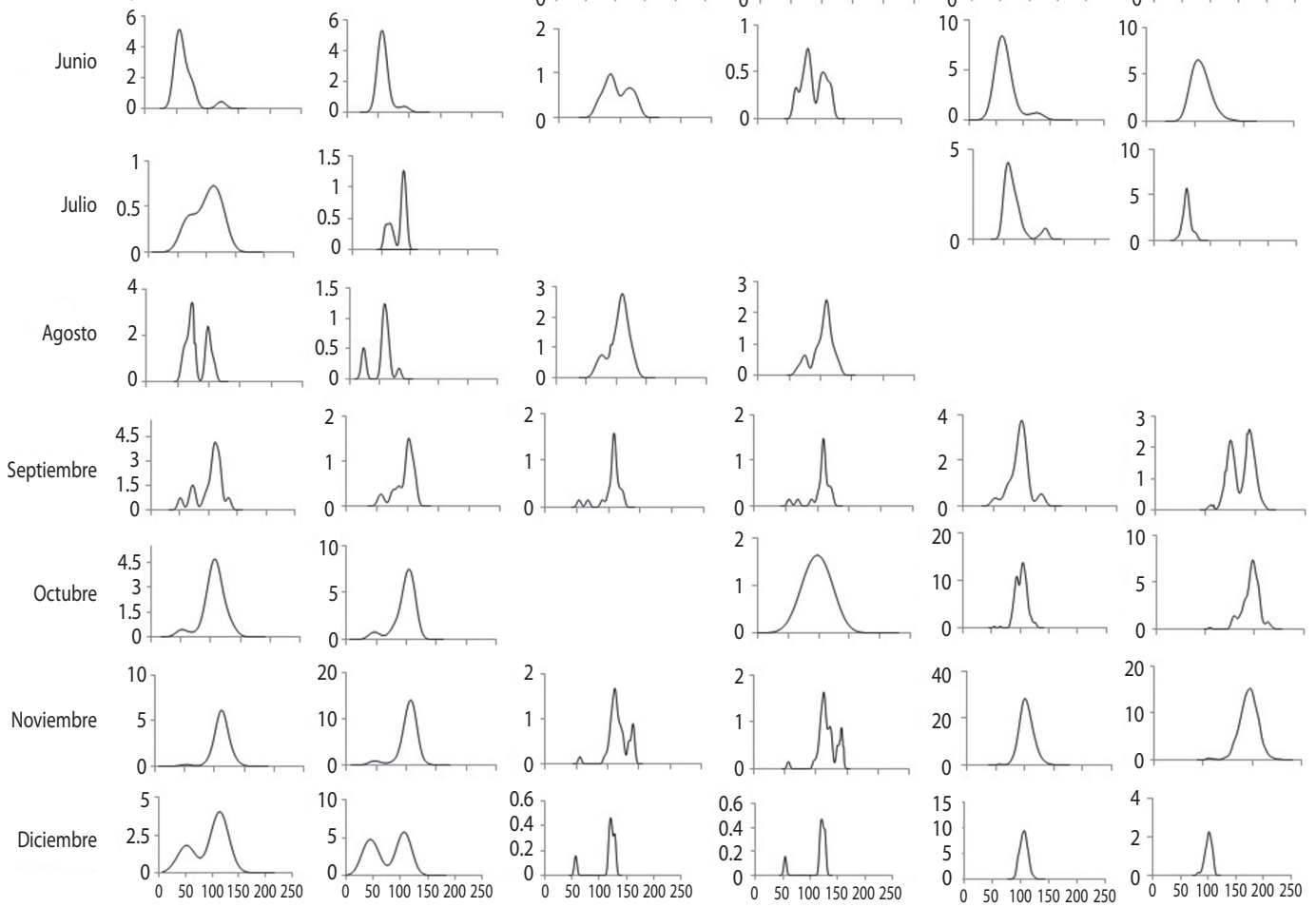

2005

2006

Hembras

2007

Fig. 4. Estructura de tallas por mes de Coryphaena hippurus, capturado por la flota artesanal en el Golfo de Tehuantepec, en el periodo 2005-2007; hembras (A) y machos (B).

Fig. 4. Monthly variations in the size structure of Coryphaena hippurus caught by the artisanal fishery in the Gulf of Tehuantepec from 2005 to 2007; females (A) and males (B). 
observó un aumento en la talla promedio para ambos sexos (Fig. 5).

\section{DISCUSIÓN}

El Golfo de Tehuantepec tiene influencia de corrientes oceánicas cálidas y la TSM mensual promedio se encuentra por arriba de $\operatorname{los} 26^{\circ} \mathrm{C}$ a lo largo del año (Magaña, Pérez, Vázquez, Carrisoza \& Pérez, 1999), esto propicia que sea una de las regiones de mayor abundancia de dorado en el Pacífico Tropical Oriental (Santana-Hernández, 2001). Los estudios de marcado en el Atlántico Norte, indican que $C$. hippurus se presenta estacionalmente en algunas áreas, con una importante sensibilidad espacial a la temperatura superficial del mar (TSM) y a la concentración de clorofila $a(\mathrm{Cl} a)$, y que además los dorados en general prefieren habitar en aguas con mucha transparencia o claridad (Farell, 2009). Así, los patrones de migración del dorado se encuentran
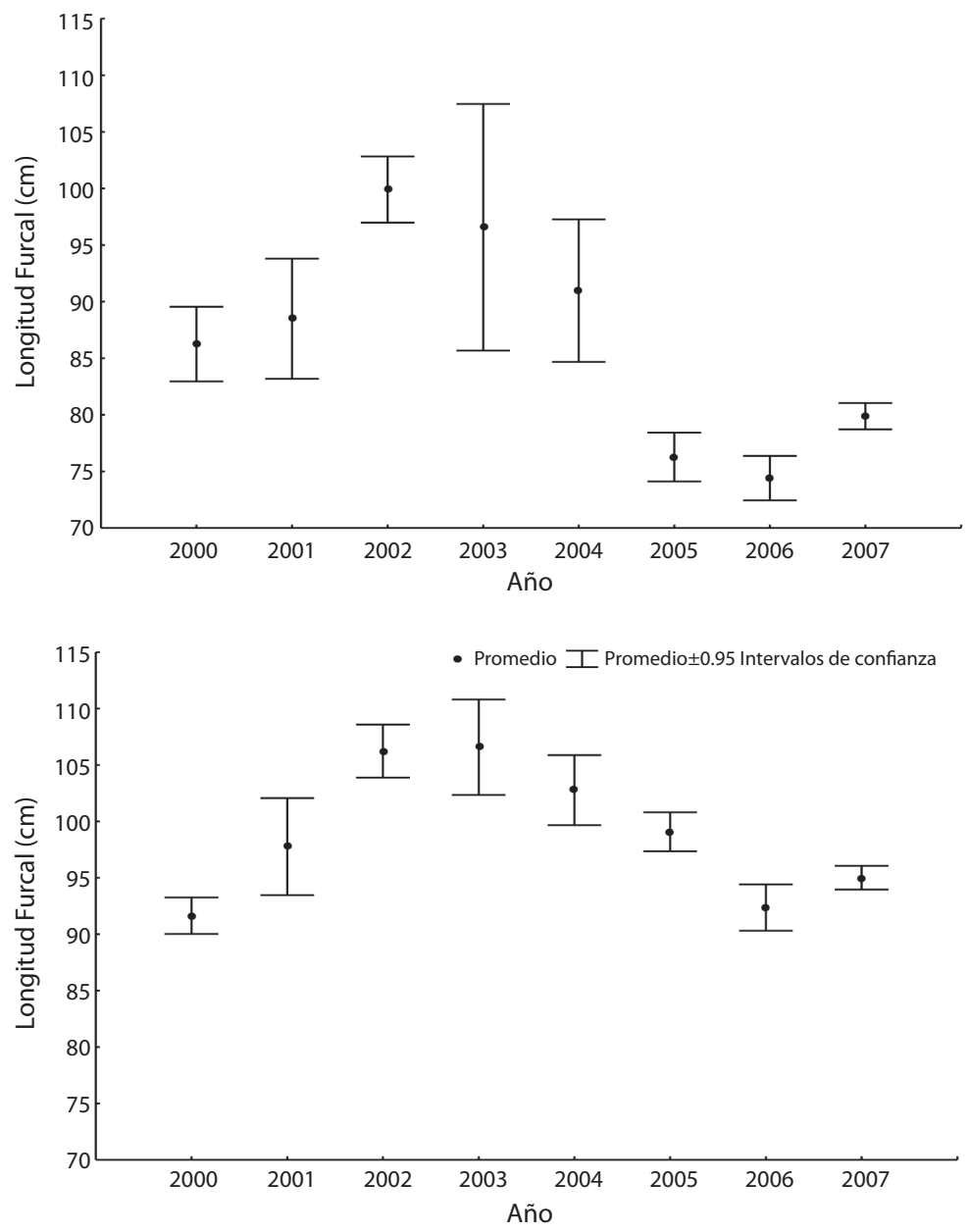

Fig. 5. Talla promedio anual para hembras (A) y machos (B) del dorado Coryphaena hippurus, capturado por la flota artesanal en el Golfo de Tehuantepec durante el periodo de 2000 a 2007. Se indica promedio (.), error estándar (barras horizontales, \pm I. C. $95 \%$ ) y tamaño de muestra (n, arriba de cada barra).

Fig. 5. Annual mean size from females (A) and males (B) of Coryphaena hippurus caught by the artisanal fishery in the Gulf of Tehuantepec from 2000-2007. Mean (.), standard error (horizontal bars, \pm I. C. $95 \%$ ) and simple size (n, above every bar) indicated. 
asociados con cambios en la TSM, que tiene un efecto sobre su distribución en el océano Pacífico (Norton \& Crooke, 1994; Norton, 1999; Santana-Hernández, 2001; Zúñiga et al., 2008; Martínez-Rincón, Ortega-García \& Vaca-Rodríguez, 2009). Por su parte Hammond (2008) en un estudio mediante marcas satelitales ha mostrado que los dorados pasan la mayor parte del tiempo en aguas superficiales cálidas $\left(27.2-29.8^{\circ} \mathrm{C}\right)$ en los $10 \mathrm{~m}$ superiores de la columna de agua.

Durante el periodo de estudio, se observó una marcada estacionalidad en las tendencias de la captura por unidad esfuerzo, principalmente en la época de surgencia, así como en la temporada de lluvias. La abundancia mensual de dorado durante el periodo de estudio siguió en términos generales las variaciones en la concentración de la $\mathrm{Cl}$ a reportadas por CervantesHernández et al. (2008). Los máximos valores sucedieron durante la temporada de Tehuanos, que corresponde con el pulso más importante de abundancia de dorado del año (de noviembre a enero).

En la temporada de lluvias (de junioseptiembre) se observó un aumento en la abundancia de dorado. Situación que pudo estar influenciada por la presencia de objetos flotantes naturales y artificiales (troncos, ramas, cuerdas, boyas y algas) que flotan a la deriva cerca de la acosta, ya que el dorado tiende a asociarse a ellos (Kojima, 1961; Rose \& Hassler, 1974). La mayoría de estos objetos flotantes son originados por el transporte de vegetación costera por las crecientes de los ríos y arroyos de temporal; así como por la apertura de las bocas de las lagunas costeras que desembocan en el mar, principalmente durante la temporada tardía de lluvias, que es además cuando se presentan los valores más altos de la biomasa de peces demersales (Tapia-García, 1998). Lo anterior, en conjunto con la dinámica ecológica de los sistemas lagunares puede contribuir a explicar la presencia del dorado cerca de la costa y por consiguiente su disponibilidad para la pesca artesanal.

Se considera que la estrecha relación que guarda el dorado con los objetos flotantes forma parte de su historia de vida. En muchas investigaciones relacionan al dorado con los objetos flotantes, ya sea como una estrategia de alimentación (Kojima, 1961; Manooch, Mason \& Nelson, 1984; Uchiyama, Bruch \& Kraul, 1986; Galván, 1999), para protegerse de sus depredadores en etapas larvales y juveniles (Rose \& Hassler, 1974) y como punto de reunión de cardúmenes o formación de estos (Freon \& Misund, 1999). No obstante que el dorado es una de las especies con mayor asociación con los objetos flotantes (Taquet et al., 2007), además de presentar las mayores capturas en los lances sobre estos (Martínez-Rincón et al., 2009), en general se sabe poco sobre este comportamiento natural (Kingsford \& DeFries, 1999; Dempster \& Kingsford, 2003).

Aunado a lo anterior, en el Golfo de Tehuantepec la variación interanual de la concentración de $\mathrm{Cl}$ a y la producción primaria (Cervantes-Hernández et al., 2008), favorecen a la producción pesquera a través de la disponibilidad y renovación de alimento, la cual modula variados procesos biológicos, tales como la reproducción, el crecimiento y el aumento en la supervivencia de los estadios larvarios (Picaut, 1985; Churchill, 2001). Cervantes-Hernández et al. (2008) registraron los menores valores en la concentración de $\mathrm{Cl} a$ entre julio y agosto, que corresponden con la canícula o sequía intraestival (Magaña et al., 1999), durante este periodo se forman giros ciclónicos/anticiclónicos que determinan en gran medida la distribución horizontal de la concentración de $\mathrm{Cl} a \mathrm{y}$ de zooplancton en el Golfo de Tehuantepec (Trasviña \& Barton, 2008). Los resultados muestran que en esta región, el dorado se encuentra disponible para la pesca artesanal durante casi todo el año, excepto de junio a agosto cuando se encuentra prácticamente ausente en las capturas artesanales.

La hipótesis del movimiento estacional de costa-mar adentro en la propuesta de Kraul (1999) y fundamentada a partir de los cambios en la condición fisiológica (FC) del dorado por Uchiyama \& Boggs (2006), puede explicar las nulas capturas en estos meses. Alejo-Plata et al. (2011) mencionan que en la zona de estudio 
los valores más altos de FC para el dorado se registraron en los pulsos de migración predesove: abril-mayo y octubre-noviembre, lo que coincidió con un aumento en las capturas costeras. Los dorados bien acondicionados entran desde el océano abierto en aguas cercanas a la costa, y participan en una intensa actividad reproductiva, lo que resulta en pérdida de peso. El dorado, ahora en un estado demacrado, regresa al mar abierto. Entre junio y agosto se presentaron los valores más bajos del FC, y el dorado prácticamente desaparece para la pesca artesanal, tal vez porque migran fuera de la costa para alimentarse y mejorar su condición durante un periodo no reproductivo. Cuando regresan a finales de septiembre e inicios de octubre, su condición se encuentra en su nivel más alto. Una evidencia de esto, es la abundancia de dorados en las capturas incidentales de la pesquería palangranera de altura frente al Golfo de Tehuantepec, durante junio a septiembre (Santana-Hernández, 2001).

Durante el periodo de estudio, los patrones temporales observados en los cambios en la proporción de sexos son consistentes con lo analizado por Alejo-Plata et al. (2011) en el Golfo de Tehuantepec, mencionan que las diferencias temporales en las capturas de machos y hembras de dorado se producen sólo durante los picos de desove; sugieren que las diferencias en la proporción de sexos son debidas al comportamiento reproductivo de la especie y no a una segregación de sexos y talla. Los machos grandes se alimentan de presas de mayor tamaño en la zona oceánica, mientras que las hembras y juveniles satisfacen sus requerimientos energéticos en la zona costera, con el consecuente ahorro de energía al no desplazarse hacia zonas oceánicas. La abundancia de machos grandes cerca de la costa durante noviembre-diciembre de los años analizados, corresponde con la temporada de desove mencionada por Alejo-Plata et al. (2011).

El aumento en la proporción de machos con la talla, ha sido observado en otras regiones geográficas (Rose \& Hassler, 1974; Massutí \& Morales-Nin, 1997; Arocha et al., 1999; Oxenford, 1999; Wu, Su \& Tawasaki, 2001).
De acuerdo a varios autores (Nakamura, 1971; Palko et al., 1982; Oxenford, 1999) la tendencia sesgada en la proporción de sexos podría deberse a una selección inadvertida de las hembras en la captura, debido a las diferentes conductas intersexuales, más que a una verdadera diferencia en la proporción de sexos o a la diferencia en la mortalidad de larvas y juveniles entre machos y hembras. Lo anterior sugiere una segregación de la población por grupos de talla y sexo, donde hembras y juveniles tienden a concentrarse preferentemente cerca de la costa, que puede constituir también una explicación de las diferencias genéticas temporales observadas por Tripp-Valdez et al. (2010).

En el análisis de la información por años se observó un ligero desplazamiento en el intervalo de tallas más frecuente, aparentemente relacionado con los años cálidos. Es posible que los cambios en la talla promedio de $C$. hippurus sean efecto de la fuerza de una clase anual. Al respecto, Alejo-Plata, Gómez-Márquez \& Salgado-Ugarte, (2011a) mencionan cuatro grupos de edad para el dorado capturado en el Golfo de Tehuantepec, el más representativo y abundante en las capturas es el grupo 1 (57 a $58 \mathrm{~cm}$ aproximadamente). Según la clave talla-edad de los organismos se observó un amplio traslape de tallas en cada grupo de edad, lo que impide observar con claridad cambios en los grupos de edad, particularmente en los de edad avanzada.

Durante 2002 a 2004 se determinaron las mayores tallas promedio para ambos sexos; las hembras se encontraron con longitudes mayores a $90 \mathrm{~cm}$ y los machos fueron mayores a $100 \mathrm{~cm}$ Lf. Durante 2005 a 2007 las tallas promedio fueron las más pequeñas para ambos sexos. No obstante la reducción de la talla promedio del dorado en el Golfo de Tehuantepec ( $90 \mathrm{~cm}$ de $\mathrm{Lf}$, talla promedio mínima estimada) no es menor que la longitud de primera madurez, que fue estimada en $48.4 \mathrm{~cm}$ de $\mathrm{Lf}$ para la hembras y $50.5 \mathrm{~cm}$ en los machos (Alejo-Plata et al., 2011) y de acuerdo con Law (2007) estas fluctuaciones no se consideran relevantes. Esto no se puede atribuir a que existan cambios en las frecuencias de tallas de las capturas. 
Por otra parte, es importante considerar los factores ambientales, así como los procesos que afectan el tamaño y la estructura poblacional de una especie (p. ej. depredación, inanición, competencia, éxito reproductivo), los cuales pueden variar considerablemente entre algunos años (Pecl, Moltschaniwskyj, Tracey \& Jordan, 2004). Además, durante el periodo 2002-2003, se registró un evento moderado "El niño" en el Pacífico (McPhaden, 2004). La manifestación de este evento podría haber provocado cambios importantes en la abundancia y estructura poblacional del dorado. En general, las hembras presentaron las menores tallas y pesos respecto de los machos, y es muy posible que esto se deba a los diferentes patrones de crecimiento por el dimorfismo sexual existente, ya que los machos crecen más rápido y alcanzan tallas y pesos mayores que las hembras (Palko et al., 1982; Massutí et al., 1999; Alejo-Plata et al., 2011a).

La distribución de tallas por mes mostró la incorporación permanente de individuos pequeños $(\leq 30 \mathrm{~cm}$ Lf) a la zona de pesca, que corresponde al reclutamiento de la población a la pesquería a lo largo del año. Al realizar el análisis de tallas por mes para $C$ hippurus mostró grupos modales similares durante el periodo de estudio. Se observaron dos cohortes principales, una de ellas antes de la temporada de lluvias (marzo-abril), y la segunda al inicio de la temporada de surgencias (noviembre). Estos resultados sugieren un reclutamiento bimodal, comportamiento reportado para las poblaciones del Golfo de California (Zúñiga et al., 2008), Pacífico norte (Kraul, 1999) y Atlántico (Massutí \& Morales-Nin, 1995).

Si se considera al reclutamiento como un proceso clave en la dinámica de una población y que los reclutas pueden ser definidos como el primer intervalo de tallas sujeto a la presión de pesca (Myers, 2002), entonces la variabilidad en la composición de tallas del dorado puede ser un buen indicador del reclutamiento.

Por lo tanto, se concluye que la estructura de tallas del dorado en el Golfo de Tehuantepec tuvo un comportamiento bimodal durante el periodo 2000 a 2007; las modas estuvieron definidas como el grupo de tamaño pequeño $(\mathrm{Lf}=50-55 \mathrm{~cm})$ y el grupo de tamaño grande $(\mathrm{Lf}=100-110 \mathrm{~cm})$. Además la variación estacional e interanual en la abundancia del dorado probablemente se encuentre relacionada con una migración pre desove, en estrecha relación con la alternancia del régimen lluvias-estiaje característico de la región y los eventos de surgencias eólicas durante la temporada de "Tehuanos". Lo anterior sugiere una separación de la población por grupos de edad, donde los individuos jóvenes con $<60 \mathrm{~cm}$ Lf tienden a encontrarse cerca de la costa, en las áreas donde la pesca artesanal incide; los organismos mayores a $100 \mathrm{~cm}$ Lf se encuentran más alejados de la costa, donde no están siendo accesibles a la pesca ribereña.

Para especies que tienen importancia recreacional y comercial es particularmente importante obtener datos actuales de su historia de vida, que pueden ser usados para asegurar una pesca sustentable. El dorado puede considerarse un recurso pesquero ideal, ya que es una especie de rápido crecimiento con importancia ecológica, comercial y recreacional, a través de su rango de distribución en el Océano Pacífico Tropical Oriental. Sin embargo, esta especie no ha recibido la atención en la recolecta de datos biológicos y evaluación que se proporciona a los grandes túnidos y peces de pico, probablemente debido al hecho de que no hay grandes pesquerías que se concentren en el dorado en esta área de estudio.

\section{AGRADECIMIENTOS}

A los pescadores de Oaxaca y Chiapas. A los compañeros del laboratorio de ictiología y biología pesquera de la Universidad del Mar por su apoyo en el trabajo de campo. A Pedro Cervantes por la revisión del documento y a los árbitros anónimos. El trabajo fue financiado por el Fondo Sectorial SAGARPA-CONACYT proyecto 225-2003 y el Gobierno del Estado de Oaxaca-CONAPESCA (2IR0502). 


\section{RESUMEN}

El dorado Coryphaena hippurus es un depredador oceánico epipelágico que se distribuye en aguas tropicales y subtropicales de todos los océanos. Son peces con alta capacidad de dispersión por su migración a gran escala. El Golfo de Tehuantepec es una de las áreas de mayor abundancia de C. hippurus, donde se registran capturas incidentales en la pesca artesanal y sostiene pesquerías recreacionales y comerciales de pequeña escala en México, Ecuador, Perú y Centroamérica. Aquí se analizan los cambios inter-anuales en la estructura de tallas por sexo del dorado durante 2000-2007, así como la tendencia de la captura por unidad de esfuerzo (CPUE) de dorado efectuada por la flota ribereña. Los resultados están basados en 3494 hembras y 3877 machos capturados por pesca artesanal en 6 localidades del Pacífico Sur provenientes de muestreos mensuales. Se estimó la CPUE a partir del número de organismos capturados por viaje de pesca. Los machos presentaron tallas entre 25.5 a $148 \mathrm{~cm}$ de longitud furcal (Lf), mientras que en hembras las tallas registradas fueron de 20.5 a $129 \mathrm{~cm}$ Lf. La proporción de sexos mostró patrones estacionales a lo largo del año y diferencias con respecto de Lf, a tallas pequeñas predominaron las hembras $(<75 \mathrm{~cm}$ FL) y a tallas grandes los machos $(>100 \mathrm{~cm}$ Lf). La estructura de Lf mostró una tendencia bimodal, con una variación en la Lf promedio de 2000 a 2007; las modas estuvieron definidas como el grupo de tamaño pequeño $(\mathrm{Lf}=50$ $55 \mathrm{~cm})$ y el grupo de tamaño grande $(\mathrm{Lf}=100 \mathrm{a} 110 \mathrm{~cm}) . \mathrm{La}$ CPUE tuvo cambios estacionales: los periodos más altos se registraron en noviembre-diciembre y mayo; los más bajos en julio-agosto. La variación estacional e interanual en la abundancia del dorado probablemente se encuentre relacionada con una migración pre desove, en estrecha relación con el régimen lluvias-estiaje característico de la región, y la temporada de surgencias eólicas asociadas a la temporada de "Tehuanos" en el Golfo de Tehuantepec.

Palabras clave: Mahi mahi, dolphinfish, pesca artesanal, estructura de tallas, CPUE, Pacífico Tropical Oriental.

\section{REFERENCIAS}

Alejo-Plata, M. C., Díaz-Jaimes, P., \& Salgado-Ugarte, I. H. (2011). Sex ratios, size at sexual maturity, and spawning seasonality of dolphinfish (Coryphaena hippurus) captured in the Gulf of Tehuantepec, Mexico. Fisheries Research, 110, 207-216.

Alejo-Plata, M. C., Gómez-Márquez, J. L., \& SalgadoUgarte, I. H. (2011a). Edad y crecimiento del dorado Coryphaena hippurus, en el Golfo de Tehuantepec, México. Revista Biología Marina y Oceanografía, 46, 125-134.

Arocha, F., Marcano, L. A., Lárez, A., Altuve, D., \& Alio, J. (1999). The fishery, demographic size structure and oocyte development dolphinfish, Coryphaena hippurus, in Venezuela and adjacent waters. Science Marine, 63, 401-409.

Arocha, F., Ortiz, M., Barrios, A., Debrot, D., \& Marcano, L. A. (2009). Catch rates for sailfish (Istiophorus albicans) from small scale fishery off La Guaira, Venezuela: period 1991-2007. Collect Volume Science Paper ICCAT, 64, 1844-1853.

Arocha, F., Ortíz, Bárrios, M., \& Marcano, L. A. (2012). Catch rates for White marlín (Tetrapturus albidus) from A.the small-scale fishery off la Guaira, Venezuela: Period 1991-2010. Collect Volume Science Paper ICCAT, 68(4), 1422-1431.

Bhattacharya, C. G. (1967). A simple method of resolution of a distribution into Gaussian component. Biometrics, 23, 115-135.

Campos, J., A. Segura, O. Lizano, \& Madrigal, E. (1993). Ecología básica de Coryphaena hippurus (Pisces: Coryphaenidae) y abundancia de otros grandes pelágicos en el Pacífico de Costa Rica. Revista Biología Tropical, 41, 783-790.

Cervantes-Hernández, P., Sánchez-Meraz, B., SerranoGuzmán, S. J., Frías-Velasco, A., Ramos-Cruz, S., \& Gracia, A. (2008). Annual abundance variation of Farfantepenaeus californiensis (Holmes 1900) in the Gulf of Tehuantepec. Hidrobiológica, 18, 215-226.

Chelton, D. B., Freilich, M. H., \& Esbensen, S. K. (2000a). Satellite observations of the wind jets off Central America. Part I: case studies and statistical characteristics. Monthly Weather Review, 128, 1993-2018.

Chelton, D. B., Freilich, M. H., \& Esbensen S. K. (2000b). Satellite observations of the wind jets off Central America. Part II: regional relationships and dynamical considerations. Monthly Weather Review, 128, 2019-2043.

Churchill, B. G. (2001). Fishery production and Mississippi river discharge. Marine Fisheries Review, 26, 17-26.

Clarke, A. J. (1988). Inertial wind path and sea surface temperatura patterns near of the Gulf of Tehuantepec and Gulf of Papagayo. Journal of Geophysical Research, 93, 12491-15501.

Collete, B. B. (1995). Coryphaenidae. In W. Fisher, F. Krupp, W. Schneider, C. Sommer, K. E. Carpenter, \& V. H. Niem (Eds.). Guía FAO para la identificación de especies para los fines de la Pesca. Pacifico Centro-Oriental, volumen II Vertebrados-Parte 1 (pp. 1036-1038). Roma: FAO.

Dempster, T. \& Kingsford, M. J. (2003). Homing of pelagic fish to fish aggregating devices (FADs): an investigation of the role of sensory cues. Marine Ecological Progress Series, 258, 213-222.

Diario Oficial de la Federación. (1995). Norma Oficial Mexicana NOM-017-PESC-1994. Para regular las actividades de Pesca deportiva en las Aguas de 
Jurisdicción Federal de los Estados Unidos Mexicanos. Tomo No. 15-19. México, D.F.

Farell, E. R. (2009). The Habitat, Movements, and Management of Dolphin, Coryphaena hippurus, in the Western North Atlantic, Caribbean, and Gulf of Mexico. (MSc Thesis). Duke University, USA.

Freon, P. \& Misund, O. A. (1999). Dynamics of pelagic fish distribution and behaviour: effects on fisheries and stock assessment. Oxford: Fishing New Books.

Galván, M. F. (1999). Relaciones tróficas interespecíficas de la comunidad de depredadores epipelágicos del océano pacífico oriental. (Ph.D. Thesis). CICESE, México.

Gallegos-García, A. \& Barberán-Falcon, J. (1998). Surgencia eólica. In M. Tapia-García (Ed.), El Golfo de Tehuantepec: el ecosistema y sus recursos (pp. 27-34). México: Universidad Autónoma Metropolitana-Iztapalapa, México.

González-Becerril, A., Espino-Barr, E. Cruz-Romero, M., \& Ruíz-Luna, A. (2000). Determinación de la unidad de esfuerzo de pesca en una pesquería artesanal ribereña en Manzanillo, Colima, México. Ciencias Marinas, 26, 113-124.

González-Silveira, A., Santamaría del Ángel, E., MillánNúñez, R., \& Manzo-Monroy, H. (2004). Satellite observations of mesoscale eddies in the Gulfs of Tehuantepec and Papagayo (Eastern Tropical Pacific). Deep-Sea Research, 51, 587-600.

Hammond, D. (2008). Using pop-off satellite archival tags to monitor and track dolphinfish and cobia. Recuperado de http://dolphintagging.homestead.com/2007Sa telliteTagCompletionRpt.pdf.

Kingsford, M. J. \& DeFries, A. (1999). The ecology and fishery of Coryphaena spp. In the waters around Australia and New Zealand. Scientia Marina, 63(34), 267-275.

Kojima, S. (1961). Studies of dolphin fishing conditions in the Western Sea of Japan. III. On the stomach contents of dolphin. Bulletin of the Japanese Society of Scientific Fisheries 27, 625-629.

Kraul, S. 1999. Seasonal abundance of the dolphinfish, Coryphaena hippurus, in Hawaii and the tropical Pacific Ocean. Scientia Marina, 63, 261-266.

Lara-Lara, J. R., Robles-Jerero, E. G., Bazán-Guzmán, M. C., \& Millán-Nuñez, E. (1998). Productividad del fitoplancton. In M. Tapia-Garcia (Ed.), El Golfo de Tehuantepec: el ecosistema y sus recursos (pp. 51-58). México: Universidad Autónoma Metropolitana-Iztapalapa.

Lasso, J. \& Zapata, L. (1999). Fisheries and biology of Coryphaena hippurus (Pisces: Coryphaenidae) in the Pacific coast of Colombia and Panama. Scientia Marina, 63, 387-399.
Lavin, M. F., Morales, J. M., Argote, M. L., Barton, E. D., Smith, R., Brown, J., Kosro, M., Traviña, A., Vélez, H. S., \& García, J. (1992). Física del Golfo de Tehuantepec. Ciencia y Desarrollo, 12, 97-108.

Law, R. (2007). Fisheries-induced evolution: Present status and future directions. Marine Ecology Progress Series, 335, 271-277.

Lluch-Cota, S., Álvarez-Borrego, E. S., Santamaría del Ángel, E. M., Mueller-Karger, F. E., \& HernándezVásquez, S. (1997). El Golfo de Tehuantepec y áreas adyacentes. Variación espaciotemporal de pigmentos fotosintéticos derivados de satélite. Ciencias Marinas, 23, 329-340.

Magaña, V., Pérez, J. L., Vázquez, J. L., Carrisoza, E., \& Pérez, J. (1999). El Niño y el clima. In V. Magaña (Ed.). Los Impactos de El Niño en México (pp. 23-67). México: Dirección General de Protección Civil, Secretaría de Gobernación.

Mahon, R. \& Oxenford, H. A. (1999). Precautory assessment and management of dolphinfish in the Caribbean. Scientia Marina, 63, 429-438.

Manooch, C. S., Mason, D. L., \& Nelson, R. S. (1984). Food and gastrointestinal parasites of dolphin, Coryphaena hippurus, collected along the southeastern and gulf coasts of the United States. Bulletin of the Japanese Society of Scientific Fisheries, 50, 1511-1525.

Martínez-Rincón, R. O., Ortega-García, S., \& Vaca-Rodríguez, J. G. (2009). Incidental catch of dolphinfish (Coryphaena spp.) reported by the Mexican tuna purse seiners in the eastern Pacific Ocean. Fisheries Research, 96, 296-302.

Massutí, E. \& Morales-Nin, B. (1995). Seasonality and reproduction of dolphinfish (Coryphaena hippurus) in the western Mediterranean. Scientia Marina, 59, 357-364.

Massutí, E. \& Morales-Nin, B. (1997). Reproductive biology of dolphinfish (Coryphaena hippurus L.) off the island of Majorca (western Mediterranean). Fisheries Research, 30, 57-65.

Massutí, E., Morales-Nin, B., \& Moranta, J. (1999). Otolith microstructure, age, and growth patterns of dolphin, Coryphaena hippurus, in the western Mediterranean. Fishery Bulletin, 97, 891-899.

McCreary, J. P., Lee, H. S., \& Enfield, D. B. (1989). The response of the coastal to strong offshore winds: with application to circulation in the Gulfs of Tehuantepec and Papagayo. Journal Marine Research, 47, 81-109.

McPhaden, M. J. (2004). Evolution of the 2002-2003 El Niño. Bulletin of the American Meteorological Society, 85, 677.

Monreal-Gómez, M. A. \& Salas de León, D. A. (1998). Dinámica y estructura termohalina. In M. TapiaGarcía (Ed.), El Golfo de Tehuantepec: el ecosistema 
y sus recursos (pp. 13-26). México: Universidad Autónoma Metropolitana-Iztapalapa.

Myers, R. (2002). Recruitment understanding densitydependence in fish populations. In P. J. B. Hart \& J. D. Reynolds (Eds.), Handbook of fish biology and fisheries (pp. 123-148). Oxford: Fish Biology, Blackwell Publishing.

Nakamura, E. (1971). An analysis of the catches and the biology of big game fishes caught by the New Orleans Big Game Fishing Club, 1966-1970. East Gulf Sport Fish. Marine Laboratory, Panama City, 38 p.

Norton, J. G. (1999). Apparent habitat extensions of dolphinfish (Coryphaena hippurus) in response to climate trasiets in the California Current. Scientia Marina, 63, 239-260.

Norton, J. G. \& Crooke, S. J. (1994). Occasional availability of dolphin, Coryphaena hippurus, to southern California Commercial Passenger Fishing Vessel Anglers: Observations and Hypotheses. California Cooperation Oceanic Fishery Investigation (CalCOFI) Report, 35, 230-239.

Oro, M. G. (1999). El Dorado (C. hippurus y C. equiselis). La Pesca deportiva en México (por la pesca libre y responsable). Recuperado de http://www.pesca.org. $\mathrm{mx} /$ articulos/dorado1.html.

Ortega-García, S., Trigueros-Salmerón, J. A., RodríguezSánchez, R., Lluch- Cota, S., \& Villalobos, H. (2000). El Golfo de Tehuantepec como un centro de actividad biológica y su importancia en las pesquerías. In $\mathrm{D}$. Lluch-Belda, J. Elorduy-Garay, S. Lluch-Cota, \& G. Ponce-Díaz (Eds.), BAC. Centros de Actividad Biológica del Pacífico mexicano (p. 335-356). México: CIBNOR.

Oxenford, H. A. (1999). Biology of the dolphinfish (Coryphaena hippurus ) in the western central Atlantic: a review. Scientia Marina, 63, 277-301.

Palko, B. J., Beardsley, G. L., \& Richards, W. J. (1982). Synopsis of biological data on dolphin-fishes Coryphaena hippurus Linnaeus and Coryphaena equiselis Linnaeus .U.S. Department Commerce, NOAA Technical Report NMFS. Circular, 443, 28 p.

Patterson, K. R. \& Martínez, J. (1991). Exploitation of the dolphin-fish Coryphaena hippurus. L. off Ecuador: Analysis by length-based virtual population analysis. Fishbyte, 9, 21-23.

Pecl, G. T., Moltschaniwskyj, N. A., Tracey, S. R., \& Jordan, A. R. (2004). Interannual plasticity of squid life history and population structure: Ecological and Management Implications. Oecologia, 139, 515-524.

Pennington, M. (1996). Estimating the mean and variance from highly skewed marine data. Fishery Bulletin, 94, 98-505.

Picaut, J. (1985). Major dynamics affecting the Eastern Tropical Atlantic and Pacific Oceans. California
Cooperation Oceanic Fishery Investigation Report, $25,41-50$.

Roden, G. I. (1961). Sobre la circulación producida por el viento en el Golfo de Tehuantepec y sus efectos sobre la temperatura superficial. Geophysical Journal International, 1, 55-76.

Romero-Centeno, R., Zavala-Hidalgo, J., Gallegos, A., \& O'Brien, J. J. (2003). Tehuantepec isthmus wind climatology and ENSO signal. Journal of Climate, 16, 2628-2639.

Rose, C. D. \& Hassler, W. W. (1974). Food habits and sex ratios of the dolphin Coryphaena hippurus captured in the western Atlantic Ocean off Hatteras, North Carolina. Transactions of the American Fisheries Society, 103, 94-100.

Rosenblatt, M. (1956). Remarks on some nonparametric estimates of a density function. The Annals of Mathematical Statistics, 27, 832-837

Salgado-Ugarte, I. H. (1992). El Análisis Exploratorio de Datos Biológicos. Fundamentos y Aplicaciones. México: Marc Ediciones y ENEP Zaragoza, UNAM.

Salgado-Ugarte, I. H., Shimizu, M., \& Taniuchi, T. (1994). Semi-graphical determination of Gaussian components in mixed distributions. Stata Technical Bulletin, $18,15-27$.

Salgado-Ugarte, I. H., Shimizu, M., \& Taniuchi, T. (1997). Nonparametric assessment of multimodality for univariate data. Stata Technical Bulletin, 38, 27-35.

Salgado-Ugarte, I. H. (2002). Suavización no paramétrica para el análisis de datos. México: Facultad de Estudios Profesionales Zaragoza y DGAPA, UNAM.

Salgado-Ugarte, I. H., J. L. Gómez-Márquez \& PeñaMendoza, B. (2005). Métodos actualizados para Análisis de Datos Biológico-Pesqueros. México: FES Zaragoza, UNAM.

Santana-Hernández, H. (2001). Estructura de la comunidad de pelágicos mayores capturados con palangre en el Pacífico mexicano (1983-1996) y su relación con la temperatura superficial del mar. (Ph.D. Thesis). Universidad de Colima, Manzanillo, Col., México.

Schwenke, K. L. \& Buckel, J. A. (2008). Age, growth, and reproduction of dolphinfish (Coryphaena hippurus) caught off the coast of North Carolina. Fishery Bulletin, 106, 82-92.

Silverman B. W. (1986). Density estimation for statistics and data analysis. London: Champan \& Hall.

Stata Corporation (2012). Stata Statistical Software: Release 10. Collage Station, TX. Stata Corporation.

Taquet, M. L., Dagorn, J., Gaertner, C., Girard, C., Riaz, A., Gorka, S., \& Itano, D. (2007). Behavior of dolphinfish (Coryphaena hippurus) around drifting FADs asnobserved from automated acoustic receivers. Aquatic Living Resources, 20, 323-330. 
Tapia-García, M. (1998). Evaluación ecológica de la ictiofauna demersal. In M. Tapia-García (Ed.). El Golfo de Tehuantepec: el ecosistema y sus recursos (pp. 103-128). México: Universidad Autónoma Metropolitana-Iztapalapa, México.

Trasviña, C. A. \& Barton, E. D. (2008). Summer circulation in the Mexican tropical Pacific. Deep Sea Research. Part. I. Oceanographic Research Papers, 55, 587-607.

Tripp-Valdez, M. A., García de León, F. J., Ortega-García, S. Lluch-Cota, D., López-Martínez, J., \& Cruz, P. (2010). Population genetic structure of dolphinfish (Coryphaena hippurus) in the Gulf of California, using microsatellite loci. Fisheries Research, 105, 172-177.

Uchiyama, J., Burch, R. K., \& Kraul, S. A. (1986). Growth of dolphins, Coryphaena hippurus and C. equiselis, in Hawaiian waters as determined by daily increments in otoliths. Fishery Bulletin, 84, 186-191.

Uchiyama, J. \& Boggs, C. (2006). Length-weight relationships of dolphinfish, Coryphaena hippurus, and wahoo, Acanthocybium solandri: seasonal effects of spawning and possible migration in the central north pacific. Marine Fisheries Review, 68 (1-4), 19-29.

Wu, C. C., Su, W. C., \& Kawasaki, T. (2001). Reproductive biology of the dolphin fish Coryphaena hippurus on the east coast of Taiwan. Fisheries Science, 67, 784-793.

Zar, J. H. (1999). Biostatistical analysis. New Jersey: Prentice Hall.

Zúñiga, M. S., Ortega-García, S., \& Klett-Traulsen, A. (2008). Interannual and seasonal variation of dolphinfish (Coryphaena hippurus) catch rates in the southern Gulf of California, Mexico. Fisheries Research, 94, 13-17. 